\section{Essentials of Maxillary Sinus Augmentation}

Publisher: Quintessence Publishing, Germany

Language: English

ISBN: 978-1-78698-018-2

Edition: 1/e

Publish Year: 2017

Pages: 128 , illustrated

Price: $78.00 €$
Authors: Francis Louise / Oana Dragan

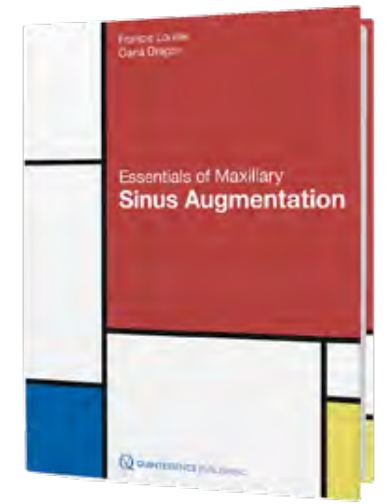

\section{Florin-Eugen}

Constantinescu

DMD, PhD Student

Holistic Dental \& Medical Institute of Bucharest-ROPOSTURO, Bucharest, Romania

e-mail:

dr.florin.constantinescu@gmail.com

It is known that most of the current work carried out by a dental practitioner goes into the preparation of the teeth for various Lately, sinus augmentation has become a current procedure in oral implantology to increase the height of the edentulous atrophied posterior maxilla. Professor Francis Louise of Aix-Marseille University, Marseille, France together with Dr. Oana Dragan of the University of Medicine and Pharmacy, Cluj-Napoca, Romania and contributors have drafted a new book to help implantologists support edentulous atrophied posterior maxilla. The book is divided in six chapters. After presenting the general considerations regarding sinus augmentation by lateral or crestal approach, the authors develop the preoperative evaluation by highlighting the necessary anatomical landmarks that the implantologist needs to know in detail. A separate chapter is dedicated to instruments and biomaterials used in oral implantology. It presents both the instruments used for the mechanical technique as well as those used for the ultrasonic one, including the effects of biomaterials on the healing of the grafted sinus. Preoperative care and procedures, surgical techniques and clinical cases are described and illustrated for the lateral and crestal techniques. At the end of the book preoperative risk assessment and postoperative care are presented. This book is updated to the currently existing knowledge, it is well illustrated with over 360 images, being a clinical guide for the implantologist to be able to plan treatment in cases with severe posterior maxillary atrophy.

DOI: 10.25241/stomaeduj.2018.5(1).bookreview.5 